\title{
Piezoelectric micromachined ultrasonic transducers with thick PZT sol gel films
}

\author{
Brahmin Belgacem • Florian Calame • Paul Muralt
}

Received: 3 March 2006 / Accepted: 5 January 2007 / Published online: 21 February 2007

(C) Springer Science + Business Media, LLC 2007

\begin{abstract}
The fabricated micro machined ultrasonic transducers (pMUT) was based on piezoelectric laminated plates operating at flexural modes. The fabricated bimorph pMUT transducers were composed of 5-layers. A $4 \mu \mathrm{m}$ thick lead zirconate titanate (PZT) thin film deposited by a sol-gel method was used. The piezoelectric layer exhibited a capacitance corresponding to a permitivity of $\varepsilon_{\mathrm{r}}=1,200$. The electromechanical coupling coefficient $\left(k^{2}\right)$ and quality factor $(Q)$ were measured as $k^{2}=4.4 \%$ and $Q=145$ in air for a low frequency transducer $(240 \mathrm{kHz})$. The effect of DC bias voltage on frequency and $k^{2}$ has been studied. The $16.9 \mathrm{MHz}$ transducer yielded values of $Q=25$ in air and $k^{2}=3 \%$.
\end{abstract}

Keywords Micromachining · Piezoelectric · Thin films · PZT

\section{Introduction}

Piezoelectricity is one of the best principles for transduction in Micro-Electro-Mechanical-System (MEMS). Indeed, piezoelectric can be used in sensors, actuators, acoustic and ultrasonic devices [1, 2]. Arrays of piezoelectric micromachined ultrasonic transducers (pMUT's) are interesting devices for ultrasonic sensors and imaging, as they offer a better impedance matching to air and water, and in addition can be fabricated with higher precision than reticulated ceramic structures [3, 4]. On the other hand, it is less obvious to reach bandwidth and sensitivity goals. In

B. Belgacem $\cdot$ F. Calame $\cdot$ P. Muralt $(\bowtie)$

Ceramics Laboratory, Ecole Polytechnique Fédérale de Lausanne (EPFL), Lausanne, Switzerland

e-mail: brahim.belgacem@epfl.ch this contribution, the questions of PZT film thickness, PZT quality, and integration possibilities are addressed.

Crucial design criteria for pMUT arrays are: (1) a high electromechanical coupling coefficient allowing for sufficiently large bandwidth, (2) limited cross coupling between elements, and (3) a good filling factor (surface of vibrating area/total area). In order to satisfy these criteria, we proposed to use both topside patterning and backside deep silicon etching to liberate the plate structures of the pMUT structures based on a SOI wafer. The principle of the chosen design is depicted in Fig. 1

The ridges on the backside, between the elements provide a stiffening structure that should give both a good rigidity of the transducer and a reduction of the cross coupling between elements.

The first part of the paper will deal with the process description. Then experimental data of long rectangular membranes will be presented.

\section{Processes}

\subsection{Process flow}

The chosen design is based on a Silicon On Insulator (SOI) wafer. A device silicon layer of $10 \mu \mathrm{m}$ is separated from the $380 \mu \mathrm{m}$ thick main body of the wafer by a $1 \mu \mathrm{m}$ thick buried oxide layer. The device layer supplies the main layer for the transducer plates, and its very uniform thickness assures a well reproducible vibration frequency of the plates. The fabrication process required 6 masks. The first four masks were used to pattern the films on the device side (front side), the other two masks define the bulk-micromachined features at the backside of the silicon wafer. The process sequence is schematically depicted in Fig. 2. 


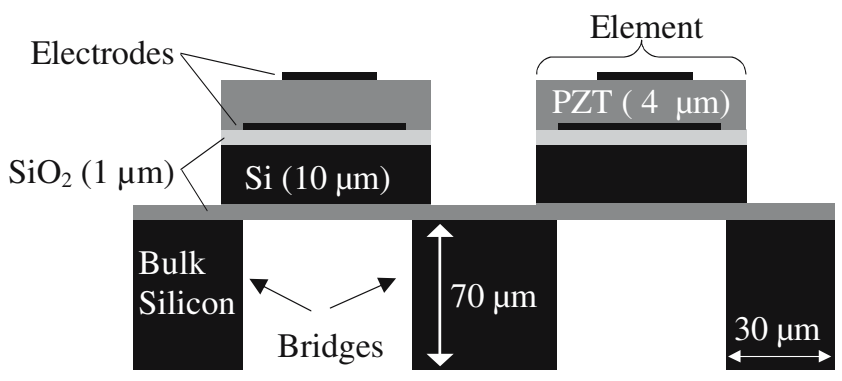

Fig. 1 Schematic drawing of a linear pMUT array

First, a $1 \mu \mathrm{m}$ wet oxide layer was grown on the $100 \mathrm{~mm}$ SOI wafer. A (111)-textured Pt film was sputter deposited and patterned by dry etching, thus obtaining the bottom electrodes. PZT sol-gel film is deposited by sol gel techniques. The platinum top electrode was sputtered and dry etched. The ridges on the backside assure a stiffening between elements and thus stop easy propagation of waves from element to element. It has been noted in addition that removal of material between the elements on the topside helps in addition to reduce cross coupling. The wet oxide on the backside was patterned for the definition of position, width and length of the ridges. The subsequent first deep silicon dry etching defined the height of the ridges. At last, the oxide mask on the ridges was removed and the membranes were liberated by a second deep dry etching while keeping the original ridge height.

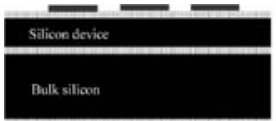

\section{Wet oxide growth}

Electrode deposition \& patterning

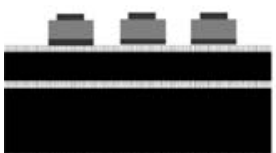

PZT Sol-gel film deposition

Top electrode deposition \& patterning

Wet etched of the PZT

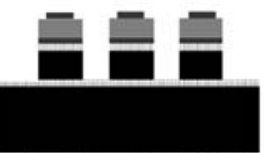

Dry etching of the oxide

Dry etching of the silicon device layer

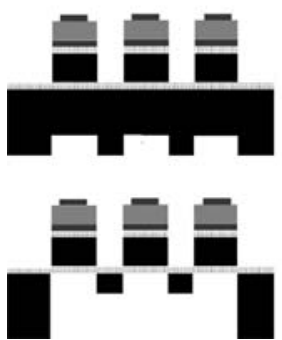

Patterning of the back wet oxide

First etching of the bulk silicon

Back wet oxide removal

\section{Etching of the bulk silicon}

Fig. 2 Process sequence for pMUT transducers

\subsection{PZT sol gel deposition}

A precursor solution of morphotropic phase boundary composition (PZT 53/47) was synthesized using an improved 2-methoxyethanol route [5] of Budd et al. [6] and Gurkovich and Blum [7]. The first step of precursor preparation was the dissolution of the lead acetate reagent in 2-methoxyethanol followed by a distillation to remove the water. The dehydrated powder is re-dissolved in dry 2methoxyethanol during $1 \mathrm{~h}$ at $110{ }^{\circ} \mathrm{C}$. Titanium and zirconium alkoxides were added and the solution was refluxed for $3 \mathrm{~h}$ at $100^{\circ} \mathrm{C}$. A vacuum distillation at $300 \mathrm{mbar}$ and $110{ }^{\circ} \mathrm{C}$ was used to remove reaction by-products and to adjust the concentration of the precursors to 0.45 molar. To improve the drying behavior of the sol-gel, 4 vol.\% formamide was added to the solution (inhibition of the hydrolysis [8]). The solution is then filtered through $0.45 \mu \mathrm{m}$ poly tetra fluoric ethylene syringe filter and stored under dry argon. Two solution precursors were prepared in

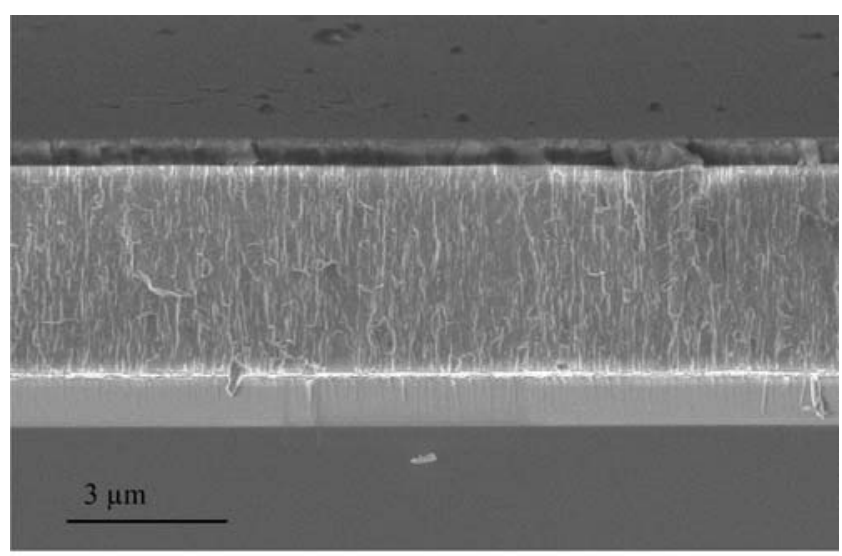

a

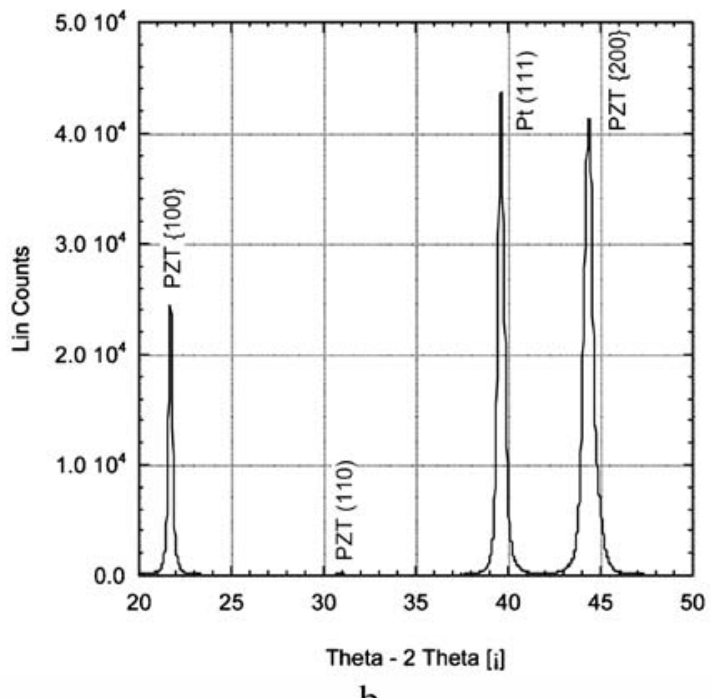

b

Fig. $34 \mu \mathrm{m}$ PZT sol-gel film on Pt electrode, a SEM cross section, b X-ray diffraction spectrum of the patterned PZT film on the device 


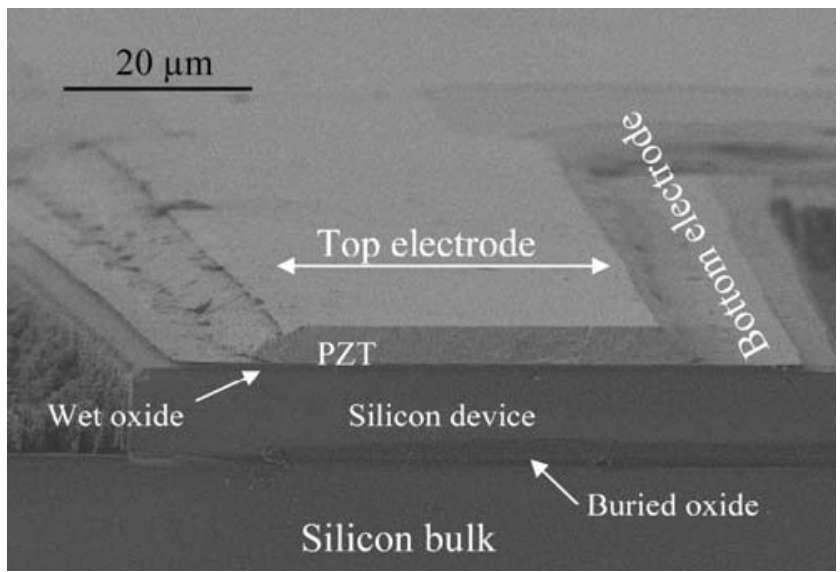

Fig. 4 Top side view of patterned transducer

order to balance the lead loss during the rapid thermal annealing (RTA). The standard one has $10 \%$ lead excess and the solution used for the last layer before the RTA has $30 \%$ lead excess. The solutions were spun on and pyrolyzed at $350{ }^{\circ} \mathrm{C}$ for $15 \mathrm{~s}$. After the deposition of four single layers (three layers with $10 \%$ lead excess, one layer with $30 \%$ lead excess), the PZT film was thermally annealed at $650{ }^{\circ} \mathrm{C}$ in oxygen for $1 \mathrm{~min}$ using a rapid thermal processing module (Process Products Corp., RTM 2016). The heating rate used in the first RTA was $30^{\circ} \mathrm{C} / \mathrm{s}$ and is then reduced to $15^{\circ} \mathrm{C} / \mathrm{s}$ for the following treatments. As film thickness of a single spin is about $60 \mathrm{~nm}, 64$ single layers were required to form $4 \mu \mathrm{m}$ PZT film. The choice of the bottom electrode is of a primary importance, as it will influence crystalline texture, quality and properties of the piezoelectric film. $\mathrm{Pt}(111)$ thin films (100 $\mathrm{nm}$ thick) grown on $20 \mathrm{~nm}$ thick $\mathrm{TiO}_{2} / \mathrm{Ti}$ adhesion layers on thermally oxidized silicon substrates have been utilized. The $\mathrm{TiO}_{2}$ layer is used to passivate partially the adhesion layer [9] in order to prevent the inter-diffusion of the Ti on top of the platinum electrode potentially modifying there the nucleation conditions [10]. As the growth of PZT is nucleated controlled [11, 12], homogeneous (111) textured platinum

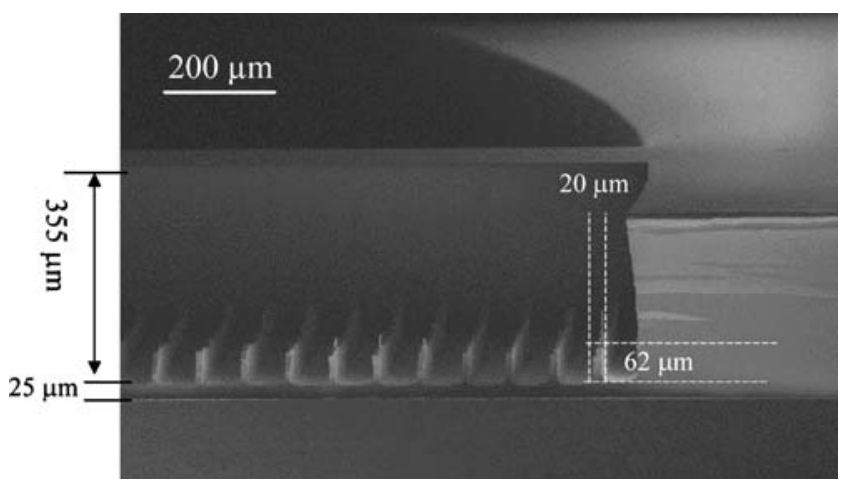

Fig. 5 Example of bulk micromachining of silicon wafer performed by deep silicon dry etching is required. The desired orientation of the PZT film, i.e., $\{100\}$ is obtained using a $20 \mathrm{~nm}$ thick $\{100\}$-oriented $\mathrm{PbTiO}_{3}[13,14]$ seed layer deposited by sol-gel.

Inspection by scanning electron microscopy (SEM) and X-ray diffraction (Fig. 3) reveal that the $4 \mu \mathrm{m}$ thick film was dense and $99 \%$ \{100\}-textured.

The high film density is also reflected in the high relative film permittivity of $\varepsilon_{\mathrm{r}}=1,200$ (at $1 \mathrm{kHz}$ ). The dielectric loss tangent amounted to $3 \%$ (at $1 \mathrm{kHz}$ ).

\subsection{Fabrication results}

The PZT film patterning was performed using a $\mathrm{HCl}: \mathrm{H}_{2} \mathrm{O}$ : HF solution at $60-65{ }^{\circ} \mathrm{C}$. Then, dry etching of both the
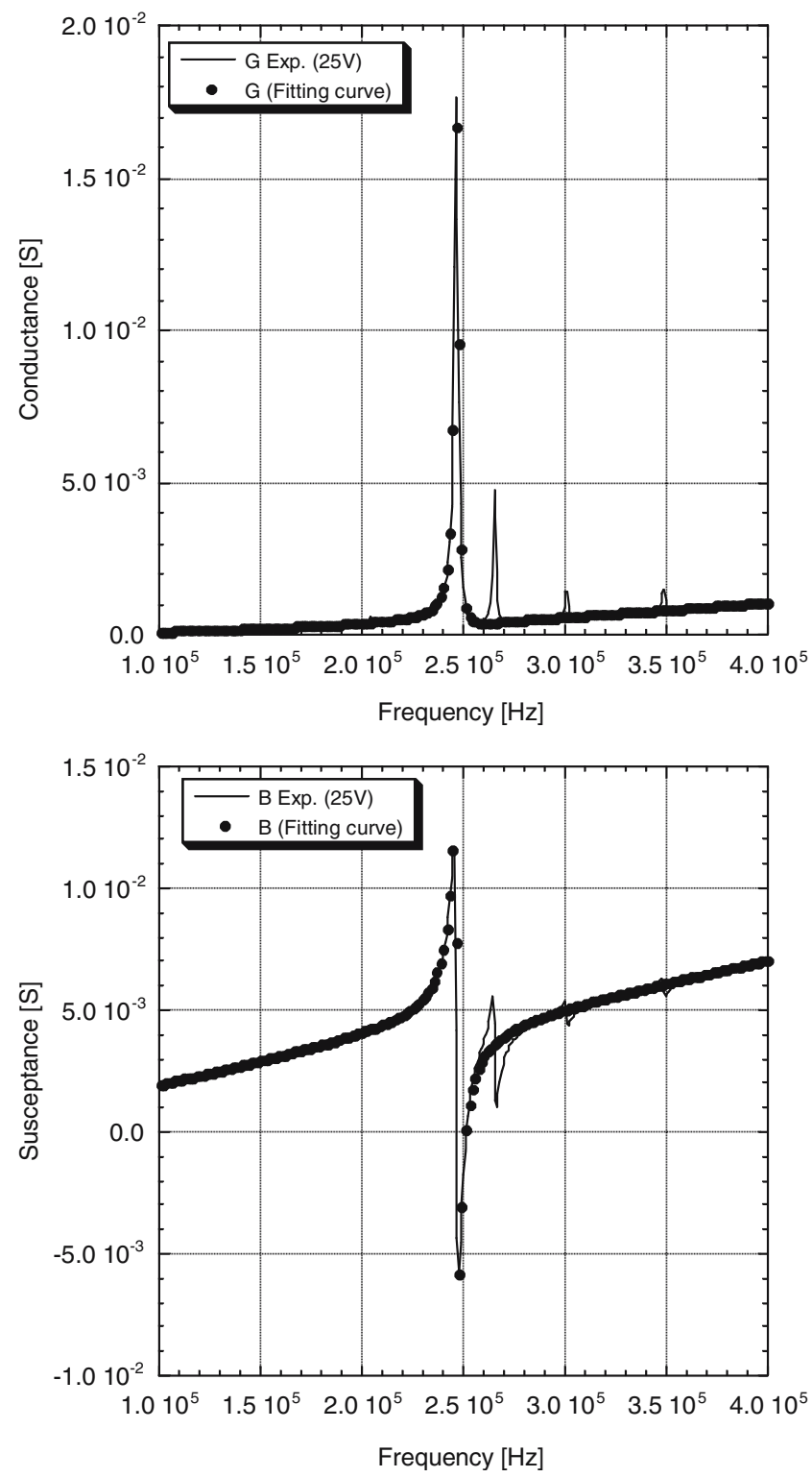

Fig. 6 Real (upper) and Imaginary (lower) part of the admittance in air (low frequency transducer) 


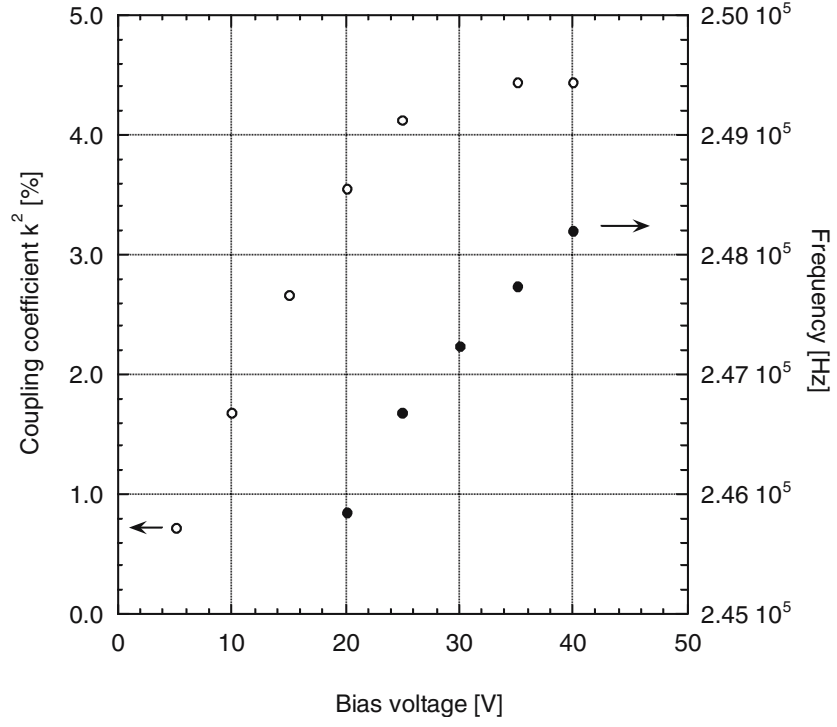

Fig. 7 Coupling factor and frequency dependency with the bias voltage in air

buried oxide and silicon device layers were performed. A SEM cross view is presented Fig. 4. This picture shows that all the etching process are well controlled. The under-etch of the PZT film is measured as around $10 \mu \mathrm{m}$ for a $4 \mu \mathrm{m}$ thick film.

However the definition of the structure on the backside of the transducer is more delicate.

Ideally, the deep silicon dry etching process should stop on the buried oxide, yielding a membrane thickness as given by the SOI wafer, and ridge dimensions of $70 \mu \mathrm{m}$ height and $30 \mu \mathrm{m}$ width. Figure 5 shows the result obtained when attempting to realize the ridges using a silicon wafer.

The method used to microstructure (two dry etching steps) the bulk silicon was found not to be homogenous and a reduction of both the width and the height during the dry etching of the ridges was observed (Fig. 5). Such difficulties are thought to be due to the non-homogenous etching rate of the structures within the wafers during the $\mathrm{BOSCH}$ process (Alcatel $601 \mathrm{E}$ ) and to the notching effect that appears when the etching species arrived on the buried oxide.

\section{Transducers characterizations}

The experimental admittance curves of single elements were measured and fitted to an equivalent circuit model (RLC) to derive both the quality factor $Q$ and the electromechanical coupling coefficient (see, e.g., [4]). Real and imaginary parts of the admittance as a function of the frequency are presented in for an applied bias voltage of $25 \mathrm{~V}$ (Fig. 6). Only an element of the low frequency transducer array was excited. Experimental and fitted curves show a good agreement. The resonance frequency of $246 \mathrm{kHz}$ agrees well with theoretical predictions given as $220 \mathrm{kHz}$. The electromechanical coupling coefficient $k^{2}$ and quality factor $Q$ were derived as $4 \%$ and 145 , respectively. The dielectric loss tangent was obtained as $2 \%$, which can be considered as a good value for a PZT thin film.

We also observed small resonances at higher frequencies, which are thought to be due to cross coupling between neighboring elements or to other vibration modes. The effect of dc bias on frequency and coupling coefficient change is shown in Fig. 7 for an element that was not previously poled.
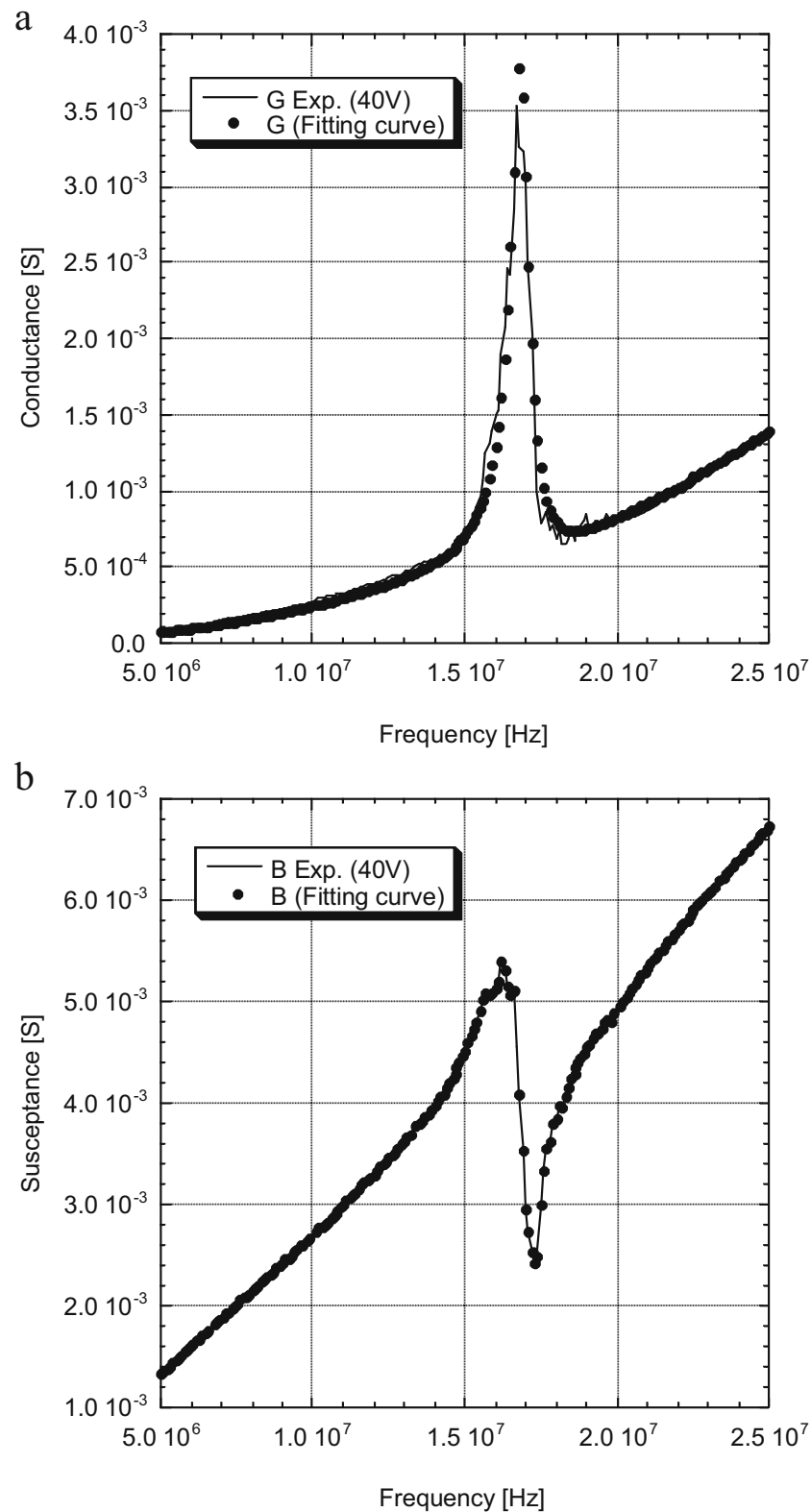

Fig. 8 Real (a) and Imaginary (b) part of the admittance in air (high frequency transducer). Solid lines: experiment; Dots: Curve fit by equivalent circuit model 
When the film is not poled, there is only a residual piezoelectric activity and the observed $k^{2}$ amounts to be less than $1 \%$. Upon application of a dc voltage, the polarization increases and so does the coupling coefficient. Saturation was reached at about $30 \mathrm{~V}$, which corresponds to a maximum of $k^{2}=4.4 \%$ for an applied field of $75 \mathrm{kV} / \mathrm{cm}$. This experimental value represents $86 \%$ of the maximum evaluated theoretical value.

The bias voltage modifies the admittance curve in various ways. First, the dc bias increases the polarization $P$ along the applied field, but at the same time it reduces the dielectric constant $\varepsilon$. The coupling coefficient is related to $P$ and $\varepsilon$ as $\left(k^{2} \propto \varepsilon P^{2}\right)$. The dc bias induces as well a tensile stress in the plane of the film by virtue of the transverse piezoelectric coupling. This stress should increase the resonance frequency, which is effectively observed in Fig. 7.

Figure 8 shows the admittance of one element within the high frequency transducer (array). The applied bias voltage was $40 \mathrm{~V}$. A resonance frequency of $16.9 \mathrm{MHz}$ was observed.

A good match between theoretical and experimental results was obtained $\left(k^{2}=3 \%, Q=25\right)$.

\section{Conclusion}

We have shown that it was possible to fabricate thick PZT sol-gel films for MEMS applications in the field of ultrasonic transducers. In order to increase the coupling coefficient of the transducer, ridges in between elements of transducers array were designed and fabricated. The topside of the pMUT transducer array was also patterned to limit the cross coupling between elements and to reduces the parasitic capacitance. Furthermore, we have demonstrated the possibility to fabricate, within the same wafer, transducers that operate at several frequencies by varying the width of the membranes.

Acknowledgment This work was supported by the Swiss Office for Education and Science (OFES) in the frame of the European project MUSTWIN, and the European project MEMSPIE. The authors would like to thank the technical staff of the center of Micro-Nano-
Technologie (CMI) of the Swiss Federal Institute of Technology (EPFL) for the clean room facilities.

\section{References}

1. P. Muralt, Ferroelectric thin films for microsensors and actuators: a review. J. Micromechanics Microengineering 10(2), 136-146 (2000)

2. S. Yuan, Z. Zhou, G. Wang, Experimental research on piezoelectric array microjet. Sens. Actuators, A 108, 182-186 (2003)

3. J.J. Bernstein, S.L. Finberg, K. Houston, L.C. Niles, H.D. Chen, L.E. Cross, K.K. Li, K. Udayakumar, Micromachined high frequency ferroelectric sonar transducers. IEEE Trans. Ultrason. Ferroelectr. Freq. Control 44, 960-969 (1997)

4. P. Muralt, N. Ledermann, J. Baborowski, A. Barzegar, S. Gentil, B. Belgacem, S. Petitgrand, A. Bosseboeuf, N. Setter, Piezoelectric micromachined ultrasonic transducers based on PZT thin films. IEEE Trans. Ultrason. Ferroelectr. Freq. Control 52(12), 2276-2288 (2005)

5. N. Ledermann, P. Muralt, J. Baborowski, S. Gentil, K. Mukati, M. Cantoni, A. Seifert, N. Setter, $\{100\}$-textured, piezoelectric $\mathrm{Pb}$ (Zrx, Ti1-x)O3 thin films for MEMS: integration, deposition and properties. Sens. Actuators, A 105, 162-170 (2003)

6. K.D. Budd, S.K. Dey, D.A. Payne, Sol-gel processing of PT, PZ, PZT and PLZT thin films. Br. Ceram. Proc. 36, 107-121 (1985)

7. S.R. Gurkovich, J.B. Blum, Preparation of monolithic leadtitanate by sol-gel process, in Ultrastructure Processing of Ceramics, Glasses and Composites, ed. by L.L. Hench, D.R. Ulrich (Wiley-Interscience, New York, 1984), pp. 152-160

8. G. Orcel, L. Hench, Effect of formamide additive on the chemistry of silica-gels, Part I, NMR of silica hydrolysis. J. Non-Cryst. Solids. 79, 177-194 (1986)

9. T. Maeder, L. Sagalowicz, P. Muralt, Stabilized platinum electrodes for PZT thin film deposition using $\mathrm{Ti}, \mathrm{Zr}$, and $\mathrm{Ta}$ adhesion layers. Jpn. J. Appl. Phys. 37, 2007-2012 (1998)

10. G.J. Willems, D.J. Wouters, H.E. Maes, R. Nouven, Nucleation and orientation of sol-gel PZT thin films on Pt electrodes. Integr. Ferroelectr. 15, 19-28 (1997)

11. K.C. Chen, J.D. Mackenzie, Crystallization kinetics of metalloorganics derived PZT thin films. Mater. Res. Soc. Symp. Proc. 180, 663-668 (1990)

12. S. Hiboux, P. Muralt, Mixed titania-lead oxide seed layers for PZT growth on Pt(111): a study on nucleation, texture and properties. J. Eur. Ceram. Soc. 24, 1593-1596 (2004)

13. T. Maeder, P. Muralt, M. Kohli, A. Kholkin, N. Setter, Pb(Zr,Ti) $\mathrm{O} 3$ thin films by in-situ reactive sputtering on micromachined membranes for micromechanical applications. Br. Ceram. Proc. 54, 206-218 (1995)

14. S. Hiboux, P. Muralt, N. Setter, Orientation and composition dependence of piezoelectric-dielectric properties of sputter deposited PZT thin films. Mater. Res. Soc. Symp. Proc. 596, 499-504 (2000) 